\title{
Template-free fabrication of hierarchical porous carbon based on intra-/inter-sphere crosslinking of monodisperse styrene-divinylbenzene copolymer nanospheres $\dagger$
}

\author{
Qingcong Zeng, ${ }^{a}$ Dingcai Wu, ${ }^{* a}$ Chong Zou, ${ }^{a}$ Fei Xu, ${ }^{a}$ Ruowen Fu, ${ }^{* a}$ Zhenghui Li, ${ }^{a}$ \\ Yeru Liang ${ }^{a}$ and Dangsheng $\mathrm{Su}^{b}$
}

Received 17th March 2010, Accepted 22nd June 2010

DOI: $10.1039 / \mathrm{c0cc00449a}$

\begin{abstract}
A novel type of hierarchical porous carbon has been successfully prepared by constructing intra- and inter-sphere - $\mathrm{CO}$ crosslinking bridges of monodisperse styrene-divinylbenzene copolymer nanospheres. The $-\mathrm{C}_{6} \mathrm{H}_{4}$ - crosslinking bridges ensure good stability of the nanospheres during swelling and crosslinking, and the -CO- crosslinking bridges play an important role in achieving good nanostructure inheritability during carbonization.
\end{abstract}

Natural species provide several examples revealing that a considerable portion of complex functionalities of living systems rely on their hierarchical structures. ${ }^{1}$ Compared to conventional porous materials consisting of uniform pore dimensions that can be adjusted over a wide range of length scales, hierarchical porous materials with well-defined pore dimensions and topologies can achieve minimized diffusive resistance to mass transport from macropores and high surface area for active site dispersion from micro- and/or mesopores. ${ }^{2}$ Without doubt, such a novel type of nanostructured materials will have played a significant role in catalysis, energy storage, adsorption, separation, etc. ${ }^{3}$ In materials science, there has been considerable interest in synthesis of such hierarchical structured materials, especially hierarchical porous carbons (HPCs). Constructing different nanoscaled pores and making them interconnected are very important in the synthesis of HPCs. To date, great achievements have been made in the preparation of well-defined HPCs by hard-/soft-templating approaches or templating and post-activation combination methods. ${ }^{4}$ For example, Cheng and co-workers prepared a 3D aperiodic hierarchical porous graphitic carbon by using $\mathrm{Ni}(\mathrm{OH})_{2} / \mathrm{NiO}$ as a hard template, ${ }^{3 b}$ and $\mathrm{Lu}$ and co-workers prepared a HPC by post-activation of Pluronic F127templated mesoporous carbon. ${ }^{4 c}$ However, most of the templates are expensive and the post-synthetic removal of the template to produce a carbon replica requires additional processing steps that are usually time-consuming, severe and harmful to the environment. These limitations impart to HPCs an uncompetitive price-to-performance ratio as compared with other materials for any given applications, and thus limit their

\footnotetext{
${ }^{a}$ PCFM Laboratory, School of Chemistry and Chemical Engineering, Sun Yat-sen University, Guangzhou 510275, P. R. China.

E-mail:wudc@mail.sysu.edu.cn(D.Wu),cesfrw@mail.sysu.edu.cn (R. Fu); Fax: + 86-020-84115112; Tel: + 86-020-84115112

${ }^{b}$ Fritz Haber Institute of the Max Planck Society, Faradayweg 4-6, D-14195 Berlin, Germany

$\dagger$ Electronic supplementary information (ESI) available: Experimental
} section. See DOI: $10.1039 / \mathrm{c} 0 \mathrm{cc} 00449 \mathrm{a}$ commercial viability. Obviously, the problem would be completely eliminated if the hierarchical porosity could be introduced into carbon without any auxiliary templates. Therefore, building controllable hierarchical porous structure through a template-free method is a great challenge for materials science.

Most recently, a template-free method to fabricate HPC has been developed by our group by constructing carbonyl (-CO-) crosslinking bridges between linear polystyrenes, where carbonyl crosslinking bridges simultaneously provided the resulting hierarchical porous polystyrene (HPP) with both a high crosslinking density and a proper amount of oxygen atoms, thus leading to good inheritability of hierarchical pore structure during carbonization. ${ }^{2}$ However, the formation and growth of network nanoparticles from crosslinking of linear polystyrene chains are hard to control very finely to some extent, and thus their shapes are not very round and their size distribution is relatively wide $(c a \cdot 10-30 \mathrm{~nm})$. This limits us to fabricating HPCs with very tunable structures.

Herein, we propose a novel template-free synthesis strategy of HPC based on intra-/inter-sphere crosslinking of monodisperse styrene-divinylbenzene (St-DVB) copolymer nanospheres with perfect spherical shape and precise size. The overall synthetic procedure is illustrated in Scheme 1. The design and construction of crosslinking bridges are the core technologies of the present method. The $-\mathrm{C}_{6} \mathrm{H}_{4}-$ crosslinking bridges from DVB ensure St-DVB copolymer nanospheres a good stability in their spherical shape during swelling and crosslinking; and the carbonyl (- $\mathrm{CO}-)$ crosslinking bridges from carbon tetrachloride $\left(\mathrm{CCl}_{4}\right)$ create the hierarchical pore structure by intra-/ inter-sphere crosslinking of polystyrene chains of nanospheres and guarantee good nanostructure inheritability during carbonization.

As is well known, conventional polystyrene nanospheres are generally obtained by microemulsion polymerization of styrene. Without doubt, such nanoparticles will dissolve in $\mathrm{CCl}_{4}$ solution and their spherical shape will be distorted seriously or even destroyed completely in the processes of swelling and crosslinking, resulting in poor controllability of pore structure. Therefore, we decided to introduce an appropriate pre-crosslinking density into polystyrene nanospheres with DVB, thus obtaining St-DVB copolymer nanospheres. The $-\mathrm{C}_{6} \mathrm{H}_{4}$ - crosslinking bridges from DVB can guarantee $\mathrm{St}-\mathrm{DVB}$ copolymer nanospheres stability in their spherical shape during swelling and crosslinking. Considering that mesopores $(2-50 \mathrm{~nm})$ result from the compact aggregation of St-DVB copolymer nanospheres, St-DVB copolymer nanospheres 


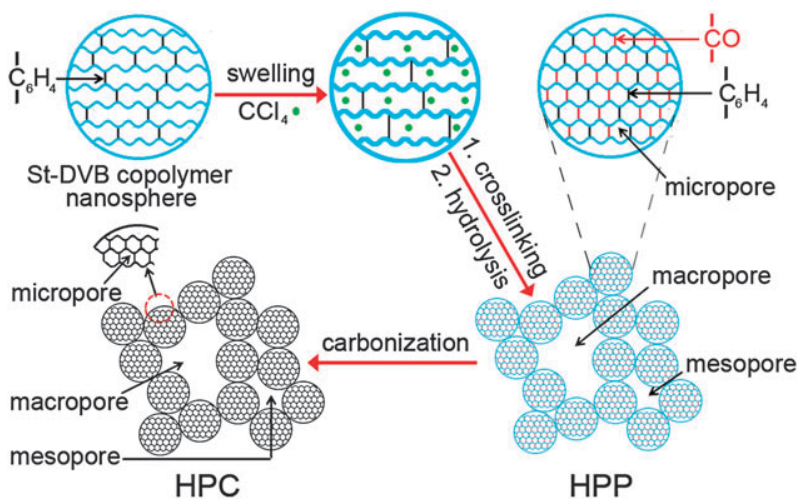

Scheme 1 Preparation mechanism of hierarchical porous polystyrene (HPP) and carbon (HPC) materials in this study.

should have appropriately small size. However, to the best of our knowledge, the size of the smallest St-DVB copolymer nanoparticles was still up to $250 \mathrm{~nm} .{ }^{5 a}$ Obviously, such large nanoparticles are not the ideal precursor for fabricating HPC, because mesopores would not be constructed effectively even if these large nanoparticles could be aggregated compactly. Therefore, much smaller monodisperse nanoparticles must be pursued. Armed with the knowledge of dispersion polymerization and the delay addition method, ${ }^{5}$ we optimized the synthesis crafts and produced successfully the smallest monodisperse St-DVB copolymer nanospheres using lower styrene nucleation concentration and more stable particles in the growing state. The diameter of nanospheres synthesized here is about $55 \mathrm{~nm}$ according to the TEM image in Fig. 1A or $60 \mathrm{~nm}$ according to dynamic light scattering (DLS) analysis in Fig. 1B, and their particle size distribution is very narrow (polydispersity index $=0.08$, Fig. $1 \mathrm{~B}$ ). It should be noted that the hydration of the hydrophilic particle shell increases the hydrodynamic radius in suspension, thus leading to a slightly higher value from DLS compared to that from TEM. ${ }^{6}$

Friedel-Crafts crosslinking reaction of the above monodisperse St-DVB copolymer nanospheres with $\mathrm{CCl}_{4}$ is induced by adding anhydrous $\mathrm{AlCl}_{3}$ to construct carbonyl (-CO-) crosslinking bridges which are essential for carbonization. ${ }^{2}$ After swelling, polymer chains inside the nanospheres are exposed completely in solvent instead of being entangled together tightly, and thus, $\mathrm{CCl}_{3}{ }^{+}$carbocations can attack the intrasphere benzene rings and form $-\mathrm{CCl}_{3}$ groups, which will further become $-\mathrm{CCl}_{2}$ - crosslinking bridges between the inner chains as the reaction proceeds. It should be noted that these $-\mathrm{CCl}_{2}-$ crosslinking bridges will be hydrolyzed into $-\mathrm{CO}-$ crosslinking bridges during washing and drying due to the presence of $\mathrm{H}_{2} \mathrm{O} .^{7}$ As a result, the intra-sphere space will be subdivided into numerous micropores by the as-constructed intra-sphere crosslinking bridges. These micropores inside the nanospheres which are shown in Fig. 1C and D can be revealed by a high magnification TEM image in Fig. 1E and a rapid uptake at low relative pressure in Fig. 2A. Moreover, according to the DFT pore size distribution (PSD) curve (Fig. 2B), these micropores concentrate at 0.6 and $1.4 \mathrm{~nm}$.

At the same time, $\mathrm{CCl}_{3}{ }^{+}$carbocations also attack the benzene rings on the surface of the nanospheres, and thus, the inter-sphere $-\mathrm{CCl}_{2}-$ crosslinking bridges will be formed

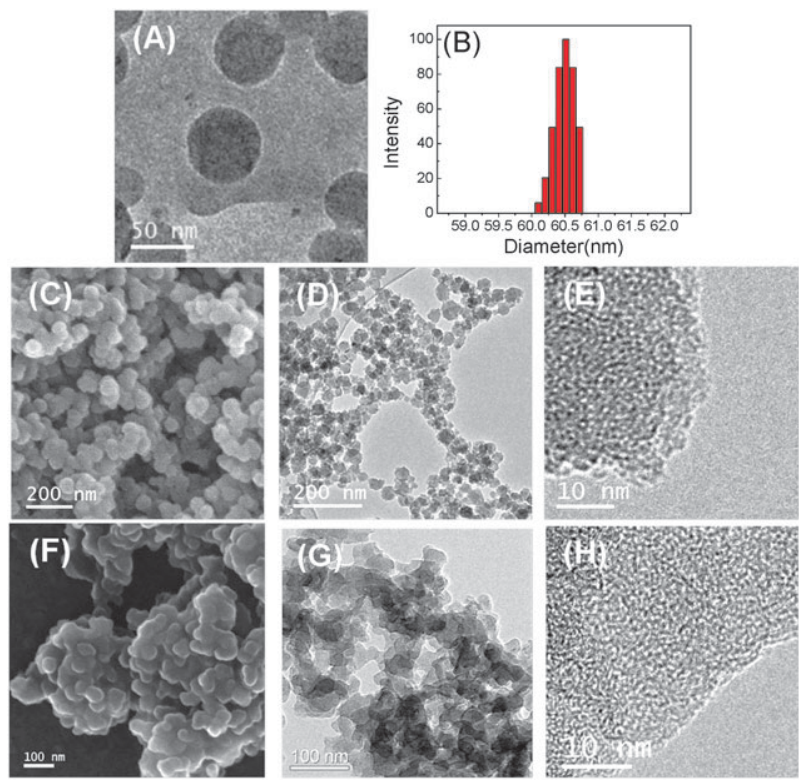

Fig. 1 (A) TEM image showing the perfect sphere shape and diameter of St-DVB copolymer nanospheres; (B) DLS particle size distribution showing the good monodispersity of St-DVB copolymer nanospheres; (C) SEM image showing the morphology of HPP; (D) TEM image showing the meso-/macropores of HPP; (E) TEM image showing the micropores of HPP; (F) SEM image showing the morphology of HPC; (G) TEM image showing the meso-/macropores of HPC; (H) TEM image showing the micropores of HPC.

and become the junctions of the nanospheres once the nanospheres stack closely in a certain orientation, eventually leading to the formation of a unique three-dimensional (3-D) network structure. As shown in the SEM image (Fig. 1C) and the TEM image in Fig. 1D, the compact and loose aggregations of nanospheres form mesopores and macropores, respectively; these meso- and macropores among the 3-D network are interconnected to each other and are also interconnected with micropores inside the network nanoparticles. The PSD curve (Fig. 2B) indicates that the length scales of meso- and macropores range from 2 to $200 \mathrm{~nm}$ and have a maximum at $40 \mathrm{~nm}$, which is determined by the St-DVB copolymer nanospheres' diameter of $55 \mathrm{~nm}$. BET calculation shows that the as-prepared HPP has a BET surface area $\left(S_{\mathrm{BET}}\right)$ of $466 \mathrm{~m}^{2} \mathrm{~g}^{-1}$; and the $t$-plot method demonstrates that the HPP's micropore surface area $\left(S_{\text {mic }}\right)$ and external (i.e., meso- and macropore) surface area $\left(S_{\text {ext }}\right)$ are 170 and $296 \mathrm{~m}^{2} \mathrm{~g}^{-1}$, respectively. Its total pore volume ( $\left.V_{\text {total }}\right)$ is measured to be $0.68 \mathrm{~cm}^{3} \mathrm{~g}^{-1}$; and micro-, meso- and macropore volumes account for $16 \%, 57 \%$ and $27 \%$, respectively, according to DFT analysis. ${ }^{8}$

The above as-prepared HPP was carbonized at $900{ }^{\circ} \mathrm{C}$, thus providing the HPC sample. Based upon SEM comparison of Fig. $1 \mathrm{C}$ and $\mathrm{F}$ and TEM comparison of Fig. 1D, E and G, H, it is found that there is no apparent difference in morphology between the samples before and after carbonization. This demonstrates that the as-constructed $-\mathrm{C}_{6} \mathrm{H}_{4}-$ and $-\mathrm{CO}-$ crosslinking bridges, especially the latter, can confer good framework carbonizability and nanostructure inheritability on the asprepared HPP during carbonization because they provide simultaneously to the HPP both a high crosslinking density and a proper amount of well-distributed oxygen atoms, 

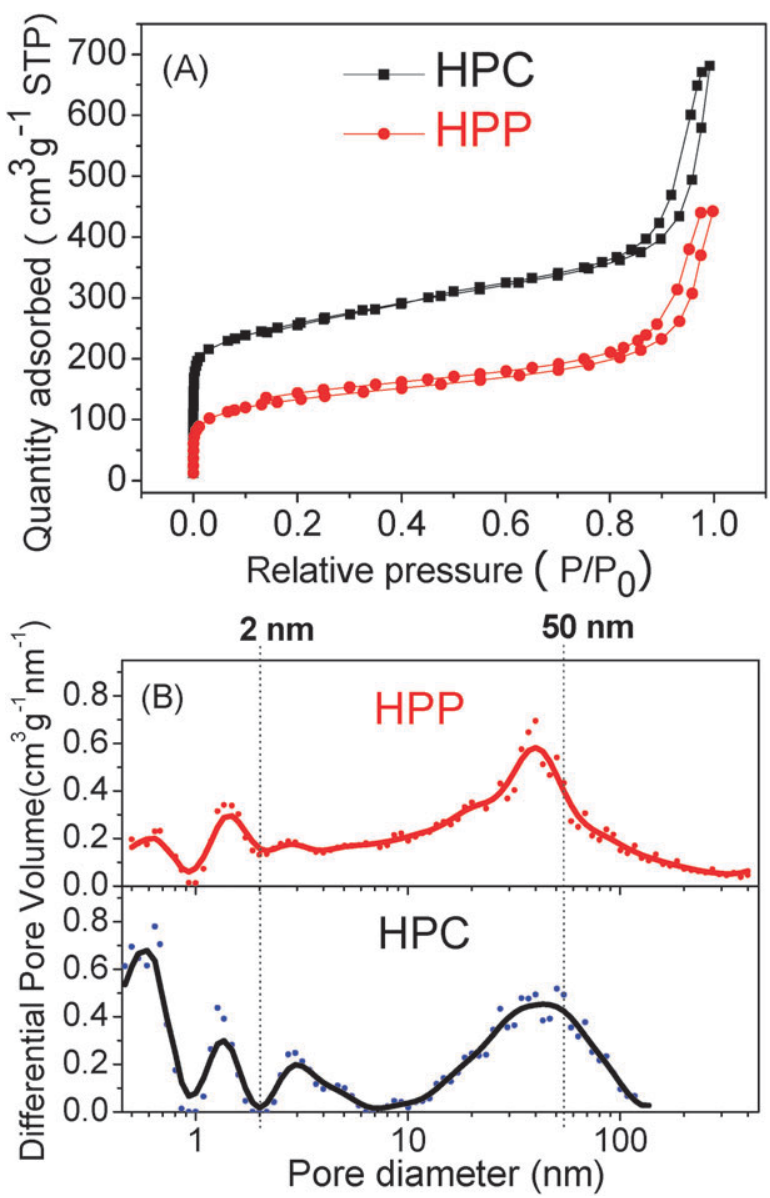

Fig. 2 (A) $\mathrm{N}_{2}$ adsorption-desorption isotherms and (B) DFT pore size distribution curves of HPP and HPC.

although they contain non-carbon elements like $\mathrm{O}$ and $\mathrm{H}$ and then fail to stay stably during the high temperature carbonization. The good nanostructure stability is also supported by the important fact that the nanosphere size-determined PSD peak of meso- and macropores at $40 \mathrm{~nm}$ still exists after carbonization (Fig. 2B). Furthermore, just like most porous carbon materials, HPC has a higher $S_{\text {BET }}\left(887 \mathrm{~m}^{2} \mathrm{~g}^{-1}\right)$ than its precursor HPP, because the emission of many non-carbon elements and carbon-containing compounds during pyrolysis will lead to the formation of numerous micropores. HPC's $S_{\text {mic }}$ and $S_{\text {ext }}$ are measured to be 444 and $443 \mathrm{~m}^{2} \mathrm{~g}^{-1}$, respectively; and its $V_{\text {total }}$ is $1.05 \mathrm{~cm}^{3} \mathrm{~g}^{-1}$, micro-, meso- and macropore volumes accounting for $20 \%, 24 \%$ and $56 \%$, respectively.
In summary, we have successfully prepared a novel type of HPC by constructing intra- and inter-sphere $-\mathrm{CO}-$ crosslinking bridges of $55 \mathrm{~nm}$ of monodisperse St-DVB copolymer nanospheres. The construction of crosslinking bridges has been proven to play a decisive role in obtaining the HPC's unique hierarchical microstructure with controllable interconnected micro-, meso- and macropores. The as-prepared HPC has a high BET surface and a large pore volume. This type of carbon nanomaterial could find lots of applications, including electrode materials in supercapacitors and fuel cells, catalysts, controlled drug delivery devices, adsorbents, and HPLC packing materials.

This research was supported by the project of NNSFC (50802116, 50632040, 50972167), the Specialized Research Fund for the Doctoral Program of Higher Education (200805581014), the Natural Scientific Foundation of Guangdong Province (8451027501001421), and the Fundamental Research Funds for the Central Universities (09lgpy18).

\section{Notes and references}

1 J. Aizenberg, J. C. Weaver, M. S. Thanawala, V. C. Sundar, D. E. Morse and P. Fatzl, Science, 2005, 309, 275.

2 C. Zou, D. C. Wu, M. Z. Li, Q. C. Zeng, F. Xu, Z. Y. Huang and R. W. Fu, J. Mater. Chem., 2010, 20, 731.

3 (a) Q. M. Ji, S. B. Yoon, J. P. Hill, A. Vinu, J. S. Yu and K. Ariga, J. Am. Chem. Soc., 2009, 131, 4220; (b) D. W. Wang, F. Li, M. Liu, G. Q. Lu and H. M. Cheng, Angew. Chem., Int. Ed., 2008, 47, 373; (c) S. Valkama, A. Nykanen, H. Kosonen, R. Ramani, F. Tuomisto, P. Engelhardt, G. T. Brinke, O. Ikkala and J. Ruokolainen, $A d v$. Funct. Mater., 2007, 17, 183; (d) J. G. Yu, Y. R. Su and B. Cheng, Adv. Funct. Mater., 2007, 17, 1984; (e) M. Hartmann, Angew. Chem., Int. Ed., 2004, 43, 5880.

4 (a) Y. Deng, C. Liu, T. Yu, F. Liu, F. Zhang, Y. Wan, L. Zhang, C. Wang, B. Tu, P. A. Webley, H. Wang and D. Zhao, Chem. Mater., 2007, 19, 3271; (b) C. Song, J. Du, J. H. Zhao, S. A. Feng, G. X. Du and Z. P. Zhu, Chem. Mater., 2009, 21, 1524; (c) W. Xing, C. C. Huang, S. P. Zhuo, X. Yuan, G. Q. Wang, D. HulicovaJurcakova, Z. F. Yan and G. Q. Lu, Carbon, 2009, 47, 1715; (d) D. C. Wu, Y. R. Liang, X. Q. Yang, Z. H. Li, C. Zou, X. H. Zeng, G. F. Lv and R. W. Fu, Microporous Mesoporous Mater., 2008, 116, 91; (e) A. Stein, Z. Y. Wang and M. A. Fierke, Adv. Mater., 2009, 21, 265; (f) Z. Y. Yuan and B. L. Su, J. Mater. Chem., 2006, 16, 663; (g) Y. S. Hu, P. Adelhelm, B. M. Smarsly, S. Hore, M. Antonietti and J. Maier, Adv. Funct. Mater., 2007, 17, 1873; (h) K. Xia, Q. Gao, J. Jiang and J. Hu, Carbon, 2008, 46, 1718; (i) J. Lee, J. Kim and T. Hyeon, Adv. Mater., 2006, 18, 2073.

5 (a) T. Gong and C. C. Wang, J. Mater. Sci., 2008, 43, 1926;

(b) J. S. Song and M. A. Winnik, Macromolecules, 2005, 38, 8300; (c) J. S. Song, F. Tronc and M. A. Winnik, J. Am. Chem. Soc., 2004, 126, 6562 .

6 J. J. Wang, Q. Li, W. Knoll and U. Jona, J. Am. Chem. Soc., 2006, 128, 15606.

7 J. Hradil and E. Králová, Polymer, 1998, 39, 6041.

8 J. Jagiello and M. Thommes, Carbon, 2004, 42, 1227. 